\title{
Profiles of Amino Acids and Acylcarnitines Related with Deltamethrin Resistance in Aedes Albopictus
}

\section{Lianfen Huang}

Guangzhou women and children's medical center

Jun Li

Southern medical university, school of public health

Xinghua Su

Southern Medical University,school of public health

Ruili Xie

Southern Medical University School of Public Health

Lilan Peng

Southern medical university, school of public health

\section{Chenfang Tang}

Guangzhou women and children's medical center

Jiabao Xu

Southern Medical University School of Public Health

Yonglan Huang

Guangzhou Women and Children's Medical Center

Hui Yan

Southern Medical University School of Public Health

Xiao-Guang Chen

Southern Medical University School of Public Health

Hua Li ( $\nabla$ smumph@163.com )

Guangzhou women and children's medical center https://orcid.org/0000-0002-2466-0084

\section{Research Article}

Keywords: Amino acids and acylcarnitines profiles, Deltamethrin resistance, Aedes albopictus

Posted Date: December 28th, 2020

DOl: https://doi.org/10.21203/rs.3.rs-135058/v1

License: () (1) This work is licensed under a Creative Commons Attribution 4.0 International License. Read Full License 


\section{Abstract}

Background: Fast emerging and increasing insecticide resistance in Asian tiger mosquito, Aedes albopictus brings alarm to the dengue epidemic over the world. The genetic and transcriptional sequencing has greatly advanced our understanding of the molecular basis for this crucial adaptation, but less is known about the regulation of insecticide resistance physiology at the metabolomic level.

Methods: We captured the relative abundance of 17 amino acids and 34 AcylCNs in the $3^{\text {rd }}$ to $4^{\text {th }}$ instar larvae of three geographically close strains of $A e$. albopictus, including a laboratory insecticide susceptible strain (Foshan) and two field deltamethrin-resistant strains (Yuexiu/Baiyun), as well as a laboratory induced deltamethrin-resistant (R18) and its parental insecticide susceptible strains (R0) to characterize their amino acids and acylcarnitines (AcylCNs) profiles by LC-MS/MS spectrometry.

Results: Heatmap and PCA analysis of amino acids and AcylCNs profile indicated a clear separation of Foshan from Yuexiu/Baiyun populations, as well as a partly overlapping between Yuexiu and Baiyun populations. All strains were abundant in five amino acids (Pro, Val, Arg, Glu, His) and two AcylCNs (C0, C2). Five amino acids (Tyr, Cit, Pro, Thr, Gly) were significantly higher, while four amino acids (His, Val, Glu, Ala) were obviously lower in Yuexiu strain than Baiyun population. R18 displayed a differential amino acid and AcylCNs profile from its parental R0 strain.

Conclusion: This study displayed a distinction of amino acids and AcylCNs profiles between insecticide-resistant and geographically or genetically-close insecticide-susceptible strain of Ae. albopictus, suggesting a possible application of amino acids and AcylCNs profiles for rapid diagnosis of deltamethrin resistance in Ae. albopictus in the future.

\section{Background}

Asian tiger mosquito, Aedes albopictus (Ae. albopictus), has spread to 70 countries all over the world out of its native home in Southeast Asia [1], with strong capacity in the transmission of various arboviruses like Dengue and Zika viruses [2]. At present, vector population control strategies, especially usage of insecticides targeting the larval and adult stages, are the most popular way to limit Ae. albopictus mosquitoes and virus transmission [3, 4]. Pyrethroids are the most frequently utilized adulticides to control the spread of Ae.albopictus to prevent dengue virus transmission as for their low mammalian toxicity and rapid knockdown effect [4-6]. However, extensive and prolonged use of pyrethroids like deltamethrin has imposed selection pressure on Ae. albopictus and eventually caused resistance [4, 7]. Fast emerging and increasing resistance to insecticides like deltamethrin in Ae. albopictus brings alarm to the dengue epidemic over the world.

Pyrethroids resistance in Ae. albopictus occurs mainly through the F1534S and F1534L mutation in the VGSC gene [7, 8], also known as knockdown resistance (kdr) resistance, as well as up-regulation of specific detoxification enzymes like glutathione S-transferase (GST) (GSTE1, AGAP009195-PA) and cytochrome P450 monooxygenases (P450s) CYP6A8, CYP9Z4, CYP6D4 [9]. Pyrethroids resistance was also regarded to be associated with pathways involved in metabolic process and pathways [10,11], protein digestion and absorption [11]. Metabolic pathways and biosynthesis of secondary metabolites have been identified to be the top-two differential pathway categories between deltamethrin-resistant and susceptible individuals of Ae. albopictus [9]. Metabolomics could reflect systematic human metabolic variations in accordance with phenotypic changes, such as resistance to insulin [12] and trastuzumab [13]. Characteristic of amino acids and AcyICNs acylcarnitine profile has become is a powerful and popular tool for diagnostic of clinical metabolic alterations [14-18]. However, little is known of the characteristic of amino acids and AcylCNs profiles in mosquitoes. Altered profile of amino acids and AcylCNs has been reported in Culex quinquefasciatus $L$ after exposure to chlorpyrifos, temephos and permethrin [19]. In this study, amino acids and AcylCNs profiles of deltamethrin-resistant and susceptible strains of Ae. albopictus were compared to observe their alterations in accordance with deltamethrin resistance.

\section{Materials And Methods}

\section{Mosquito strains, analysis of insecticide resistance and kdr mutations}

Larvae of two field strains of Ae. albopictus were collected from public land like parks, schools and residential areas in 2017 in two districts named Yuexiu and Baiyun of Guangzhou city respectively, with dengue incidence of $>5$ and $1-5$ cases per 100,000 people respectively as previous report [7]. The larvae were housed [7] and have been tested for their larval resistance to four insecticides: (1) organophosphate, temephos; (2) microbial bacterial toxin, Bti; (3) hormonal insect growth regulators, pyriproxyfen (PPF); (4) the chitin biosynthesis inhibitor, hexaflumuron; as well as their adult resistance to five adulticides including deltamethrin, permethrin, malathion, bendiocarb, DDT following WHO guidelines [7]. The larvae housed from both Yuexiu and Baiyun districts were also tested for their resistance to adulticides including deltamethrin, permethrin, DDT, bendiocarb, malathion following WHO guidelines as previous report [7, 20]. For larval bioassays, the resistant status was evaluated by the resistant ratio $\left(R_{50}\right)\left(R R_{50}<5\right.$, susceptible; $5<R R_{50}<10$, moderately resistant; $R R_{50}>10$, highly resistant), for adult bioassays, resistant status was defined by mortality rate: resistant if mortality $<90 \%$, probably resistant if mortality was between 90 and $98 \%$, and susceptible if mortality $>98 \%$ [7]. The kdr mutation in resistant and susceptible individuals from Yuexiu and Baiyun districts in 2017 has been genotyped at the voltage-gated sodium channel (VGSC) gene after exposure to deltamethrin, permethrin, and DDT to detect mutations within domains II, III and IV [7], samples exposed to bendiocarb have been genotyped at the ace-1 gene to detect mutations within G119 [7]. The Foshan strain of Ae. albopictus, an insecticide-susceptible stain which was reared in laboratory without exposure to any insecticides since its collection in 1983 in Foshan (a smaller city adjacent to Guangzhou city), was used as the control strain for metabolomic comparison with these field strains of Ae. albopictus.

$\mathrm{R} 18$ is a laboratory resistant strain $\left(\mathrm{LC}_{50}=0.039, \mathrm{RR}_{50}=13\right)$ after deltamethrin selection of an insecticide susceptible Ae. albopictus populations from Shanghai CDC for 17 generations, which was primarily screened by $0.005 \mathrm{mg} / \mathrm{L}$ of deltamethrin (Sigma, USA) from the 1 st to the 10 th generation, followed by 
deltamethrin screening at $50 \%$ lethal concentration $\left(\mathrm{LC}_{50}\right)$ from the 11 th to the 22 nd generation as described previously [21]. The parental strain of $A e$. albopictus without any exposure to insecticides (R0) was used as an insecticide-susceptible control strain for R18 strains of Ae. albopictus in this study, $\mathrm{LC}_{50}$ $(95 \%)=0.003(0.001,0.006) \mathrm{mg} / \mathrm{L}$. The larval and adult resistance of R18 to deltamethrin was also analyzed following WHO guidelines as previous report [7, 20]. The resistant and susceptible adults against deltamethrin from R18 strains of Ae. albopictus were also genotyped for kdr mutation as previous report [7].

\section{LC-MS/MS sample preparation and metabolites extraction}

Six replicates of ten numbers of 3rd to 4th instar larvae (approximately $2 \mathrm{mg} / \mathrm{larvae}$ ) of Foshan, Yuexiu, Baiyun, R0, R18 strains were sampled respectively. Then, they were homogenized (TIANGEN OSE-Y10-plus) in $500 \mu \mathrm{l}$ of $\mathrm{ddH}_{2} \mathrm{O}$, followed by centrifugation at $4{ }^{\circ} \mathrm{C}, 13000 \mathrm{rpm}$ for $10 \mathrm{~min}$. The lysate was transferred to a sterile syringe which was attached to a $0.22 \mu \mathrm{m}$ acrodisc for filtration. Finally, $30 \mu \mathrm{l}$ of the filtered lysate was added to the filter paper (S\&S903) and dried for storage. A paper circle with $3.2 \mathrm{~mm}$ diameter was punched from the dry larval lysate filter paper by a Panthera-Puncher TM 9 Puncher (PerkinElmer, Waltham, MA, USA)]. A NeoBase non-derivatized MS/MS kit (Perkin Elmer) was employed for extraction of amino acids and AcylCNs. Metabolomics analysis

LC-MS/MS (API 2000, ABSciex, Framingham, MA, USA) platform, with stable isotopes labelled amino acid standards (Cambrige Isotope Labs) as an internal solution, was selected for quantification of 17 amino acids including alanine, arginine, aspartate, citrulline, glutamine, glutamate, glycine, histidine, leucine, methionine, ornithine, phenylalanine, proline, serine, threonine, tyrosine, valine) and 34 AcylCNs including CO, C2, C3, C3DC, C4, C4-OH, C4DC, C5, C5:1, C5-OH, C5DC, C6, C6:1, C6DC, C8, C8:1, C10, C10:1, C10:2, C12, C12:1, C14, C14-OH, C14:1, C14:2, C16, C16:1, C16-OH, C16:1-OH, C18, C18:1, C18-OH, C18:2, C18:1-OH (34AC). Analysis of 17 amino acids and 34 AcylCNs was interpreted in multiple reaction monitoring (MRM) mode with Analyst 1.6 .2 software (ABSciex) and NeoBase database.

\section{Statistical analysis}

Finally, the contents of 17 amino acids and 34 AcylCNs were scaled according to the preparation procedure of dry larval lysate filter paper. The concentration of the detected metabolites was normalized and analyzed by orthogonal pattern recognition methods principal component analysis (PCA). PCA with performed using Metaboanalyst (v3.0) (https://www.metaboanalyst.ca/faces/home.xhtml) to display the characteristic of amino acids and AcylCNs profiles of Ae. albopictus with different levels of deltamethrin resistance. SPSS 17.0 was applied for the spearman correlation analysis between the deltamethrin $\mathrm{LC}_{50}$ values and the contents of amino acids and AcylCNs in Ae. albopictus.LC ${ }_{50}$ and $\mathrm{KDT}_{50}$ were estimated using the log-probit models [7]. For larvae bioassays, the resistant status was measured by the resistant ratio $\left(R R_{50}\right)$, i.e., the ratio of $L C_{50}\left(\right.$ or $\left.I E_{50}\right)$ for the field population over $L C_{50}$ (or $\left.I E_{50}\right)$ for the laboratorysusceptible strain [7]. Larval resistance status was defined as susceptible if $R R_{50}<5$, moderately resistant if $5<R R_{50}<10$, and highly resistant if $R R_{50}>10$ [7]. For adult bioassays, resistant status was defined by mortality rate: Resistant if mortality $<90 \%$, probably resistant if mortality was between 90 and $98 \%$, and susceptible if mortality $>98 \%[7,9]$. The relationship between nonsynonymous mutations and resistance was verified by Fisher's exact test or the $\chi 2$-test (when all $n>5$ ), and the odds ratio (OR) was calculated for each mutation [7].

\section{Results}

Insecticide resistance and kdr mutation of Ae. albopictus larvae from Yuexiu and Baiyun districts in 2017

Ae. albopictus larvae from both Yuexiu and Baiyun districts had moderate to high resistance to adulticides like deltamethrin and permethrin, both of them remained susceptible to malathion (Table 1). Moreover, larvae from Yuexiu district had developed much higher level of resistance to deltamethrin than the larvae from Baiyun district, but larvae from Baiyun district was more resistant to both permethrin and DDT (Table 1). In 2017, the larvae and adult populations of Ae. albopictus from Baiyun/Yuexiu districts of Guangzhou city, China has developed high resistance to currently used insecticides (deltamethrin, permethrin, DDT and bendiocarb, mortality < 90\%) except malathion (mortality > 98\%) [7]. The mortality rate against deltamethrin, permethrin, DDT and bendiocarb was 5.6\%, 55.2\%, 52.1\%, 42.4\% in the adult populations from Yuexiu district, and 38.4\%, 68.8\%, 74.7\% and 51.2 in the adult population from Baiyun district, respectively [7]. Ae. albopictus larvae from both districts were still sensitive to Bti and hexaflumuron ( $\left.\mathrm{RR}_{50}<5\right)$, but high resistance to pyriproxyfen $\left(\mathrm{RR}_{50}>10\right)[7]$.

Table 1

The larval resistance of Ae. albopictus from both Yuexiu and Baiyun districts to five adulticides (deltamethrin, permethrin, DDT, bendiocarb, malathion) in 2(

\begin{tabular}{|c|c|c|c|c|c|c|c|c|c|}
\hline & \multicolumn{2}{|l|}{ deltamethrin } & \multicolumn{2}{|l|}{ Permethrin } & \multirow{2}{*}{$\begin{array}{l}\text { DDT } \\
\text { LC }_{50}(95 \%)(\mathrm{mg} / \mathrm{L})\end{array}$} & \multicolumn{3}{|c|}{ Bendiocarb } & \multirow{2}{*}{$\begin{array}{l}\text { Malathion } \\
\mathrm{LC}_{50}(95 \%)(\mathrm{mg} / \mathrm{L})\end{array}$} \\
\hline & $\mathrm{LC}_{50}(95 \%)(\mathrm{mg} / \mathrm{L})$ & $\mathrm{RR}_{50}$ & $\mathrm{LC}_{50}(95 \%)(\mathrm{mg} / \mathrm{L})$ & $\mathrm{RR}_{50}$ & & $\mathrm{RR}_{50}$ & $\mathrm{LC}_{50}(95 \%)(\mathrm{mg} / \mathrm{L})$ & $\mathrm{RR}_{50}$ & \\
\hline Baiyun & $0.027(0.020,0.049)$ & 27 & $0.628(0.527,0.730)$ & 15 & $5.281(5.073,5.538)$ & 12.1 & $1.460(0.995,1.745)$ & 0.77 & $0.073(0.068,0.080)$ \\
\hline Yuexiu & $0.066(0.061,0.094)$ & 66 & $0.356(0.113,0.533)$ & 8.47 & $1.912(1.560,2.227)$ & 4.37 & $5.37(4.826,6.031)$ & 5.54 & $0.092(0.081,0.109)$ \\
\hline Foshan & $0.001(0.001,0.006)$ & 1 & $0.042(0.031,0.053)$ & 1 & $0.438(0.389,0.484)$ & 1 & $0.969(0.908,1.038)$ & 1 & $0.043(0.039,0.046)$ \\
\hline
\end{tabular}

Kdr mutation associates with deltamethrin resistance in R18 strain

The larvae and adult populations of Ae. albopictus from R18 has developed high resistance to deltamethrin (larval $\mathrm{LC}_{50}=0.039, \mathrm{RR}_{50}=13$, adult mortality $=$ $85 \%)$. Sequences of domains II (480 bp), III (346 bp) and IV (280 bp) of the VGSC gene were also obtained from the resistant and susceptible mosquitoes after deltamethrin adult bioassays for R18 [7]. Kdr genotyping showed non-synonymous mutations of F1534S could be detected at codon 1534 in domain III of 
R18, where wild-type TTC (Phe) was changed to TCC (Ser) at a high frequency of 78.38\% (29/37), indicating a significant correlation with deltamethrin resistance (Table $2, \mathrm{p}<0.01)$.

Table 2

Kdr mutations at position F1534 of the VGSC gene in adults of Ae. albopictus from R18 strain and its parental susceptible RO strain.

\begin{tabular}{|llllllllll|}
\hline Insecticide & N & \multicolumn{3}{c|}{ Genotype } & & \multicolumn{5}{l|}{ Odds Ratio(95\%Cl) } & \\
\cline { 3 - 11 } & & FF & FS & SS & FL & LL & F1534S & F1534L \\
\hline Susceptible strain (R0) & 40 & 40 & 0 & 0 & 0 & 0 & 1 & - \\
\hline R18 & 37 & 9 & 23 & 6 & 0 & 0 & $123.00(14.30,1013.39) * *$ & - \\
\hline$* *: p<0.01$ & & & & & & & & \\
\hline
\end{tabular}

Differential larval amino acids and AcyICNs profiles between insecticide-resistant Ae. albopictus from Baiyun/Yuexiu and susceptible Foshan strain

Amino acids and AcylCNs profile analysis demonstrated that Pro, Val, Arg, Glu and His were the five most abundant amino acids (Fig. 1A) whereas C0, C2 were the richest AcylCNs in the larvae of Ae. albopictus (Table S2) \& (Figs. 1B and 1C). The metabolites in Baiyun/Yuexiu larvae displaced fluctuation in comparison to Foshan larvae. T test analysis showed the concentration of five amino acids Tyr, Cit, Pro, Thr, Gly (Fig. 2A) and five AcylCNs C3DC, C6-1, C10, C10-1,C10-2 (Fig. 2B) were significantly higher, while the levels of C2 (Fig. 2B) and four amino acids His, Val, Glu, Ala (Fig. 2A) were obviously lower in Yuexiu strain in comparison to Foshan strain. Similarly, Cit and Arg were increased while His was decreased in Baiyun strain (Fig. 2C). Heatmap and PCA analysis of 17 amino acids and 34 AcylCNs contents indicated significant discrepancy of amino acids and AcylCNs profile between Foshan and Baiyun/Yuexiu larvae (Figs. 3A and 3B). However, profile of Yuexiu larvae was partly overlapped with that of Baiyun larvae (Fig. 3B).

\section{Differential larval amino acids and AcylCNs profiles between deltamethrin-resistant (R18) and its parental susceptible (R0) strains}

Amino acids and AcyICNs profile analysis demonstrated that Pro, Val, Arg, Glu and His were the five most abundant amino acids whereas C0, C2 were the richest AcylCNs in the deltamethrin-resistant (R18) and -susceptible (R0) larvae of Ae. albopictus (Table S2). Between deltamethrin-resistant R18 and susceptible (R0) strains, no significant difference of amino acids and AcylCNs were found, but the heatmap and PCA analysis of 17 amino acids and 34 AcylCNs contents indicated discrepancy of amino acids and AcylCNs profile (Figs. 4A and 4B).

Table 3

Kdr mutations at position F1534 of the VGSC gene in resistant and susceptible adults of Ae. albopictus against deltamethrin, permethrin and DDT from both Yuexiu and Baiyun districts in 2017 [7]

\begin{tabular}{|c|c|c|c|c|c|c|c|c|c|}
\hline \multirow[t]{2}{*}{ Insecticide } & \multirow[t]{2}{*}{ Phenotype } & \multirow[t]{2}{*}{$\mathbf{N}$} & \multicolumn{5}{|c|}{ Genotype } & \multicolumn{2}{|l|}{ Odds Ratio(95\% Cl) } \\
\hline & & & FF & FS & SS & FL & LL & F1534S & F1534L \\
\hline \multirow[t]{2}{*}{ Deltamethrin } & $\mathrm{S}$ & 20 & 17 & 3 & 0 & 0 & 0 & 1 & 1 \\
\hline & $\mathrm{R}$ & 41 & 2 & 1 & 17 & 3 & 18 & $4.44(1.12,17.52) *$ & NA \\
\hline \multirow[t]{2}{*}{ permethrin } & $S$ & 19 & 11 & 5 & 0 & 1 & 1 & 1 & 1 \\
\hline & $\mathrm{R}$ & 42 & 1 & 4 & 22 & 5 & 11 & $4.55(1.38,15.05)^{\star}$ & $27.20(4.60,160.69)^{\star \star}$ \\
\hline \multirow[t]{2}{*}{ DDT } & S & 18 & 15 & 2 & 1 & 0 & 0 & 1 & 1 \\
\hline & $\mathrm{R}$ & 44 & 5 & 7 & 15 & 11 & 6 & $5.00(1.27,19.74)$ & $N A^{\star \star}$ \\
\hline
\end{tabular}

\section{Discussion}

For insecticide resistance monitoring, the bioassays like WHO susceptibility test [22] and CDC bottle assay [23], molecular detection of knock-down resistant gene mutations, analysis of metabolic enzymes GSTs, p450s and CCEs activities have been frequently used, but limitations existed, such as time and laborconsuming, difficult for standardization with complicated procedures, requirement of special insectary laboratory to rear mosquitoes and subjective judgement of larval and adult death, etc. Amino acids and AcyICNs profile have been popular for diagnostic of clinical metabolic diseases. In this study, we first tried to explore the characteristic of amino acids and AcyICNs profile among geographically or genetically-close strains of Ae. albopictus with differential resistance to insecticides especially deltamethrin, and compared their differences. The insecticide resistance status and the corresponding possible genetic mechanism of the test strains was analyzed thoroughly before their metabolomic analysis. Deltamethrin was the most popular adulticide for vector control in urban areas of Guangzhou city [7]. Accordingly, the most significant difference of insecticide resistance between the larvae and adults from different districts were their differential resistance to deltamethrin, such as significantly higher larval and adult resistance to deltamethrin in Yuexiu strain (larval $R_{50}=66$, adult mortality of $5.6 \%$ ) than Baiyun strain (larval $\mathrm{RR}_{50}=27$, adult mortality of $38.4 \%$ ) (Table 1) [7]. In Ae. albopictus adults from both districts, the resistance to pyrethroids (deltamethrin, permethrin) and DDT has been proved to be significantly associated with non-synonymous 1534 codon mutations from homozygote wild-type TTC (Phe) (F1534F) to homozygote TCC (Ser) (F1534S) or CTC (Leu) (F1534L) to at domain III (346 bp) of the VGSC gene, with five detected kdr genotype (FF, FS, SS, FL, LL) in the resistant and susceptible individuals of Ae. albopictus adults for three insecticides (deltamethrin, permethrin and DDT) from both districts (Table 3) [7] as previous report [8]. Similarly, Kdr genotyping detected F1534S mutation at codon 1534 in domain III of R18 strain 
at a frequency of $78.38 \%$ (29/37), indicating a significant correlation of F1534S mutation with laboratory-induced deltamethrin resistance in Ae. albopictus (Table 2, p < 0.01).

The PCA analysis of amino acids and AcylCNs profiles among different strains demonstrated that Ae. albopictus larvae with differential deltamethrin resistant level displaced differential amino acids and AcyICNs profiles, including clear distinction of amino acids and AcylCNs profile between insecticide-susceptible larvae (Foshan strain) and larvae collected from geographically-close and insecticide-resistant Baiyun/Yuexiu districts, or obvious difference between a laboratory-induced deltamethrin-resistant strain (R18) and its parental susceptible Shanghai strain (R0), whereas it was relatively similar between insecticideresistant larvae from relative geographically-close Baiyun and Yuexiu districts. This may suggest a potential role of amino acids and AcylCNs profile in predicting larval insecticide resistance of Ae. albopictus.

Moreover, five amino acids (Tyr, Cit, Pro, Thr, Gly) were significantly higher, whereas four amino acids (His, Val, Glu, Ala) were obviously lower in larvae from Yuexiu district than the susceptible Foshan strain. However, only Cit and Arg were increased while His was decreased in larvae from Baiyun district than the susceptible Foshan strain, this phenomenon was probably due to their differential resistance to insecticides especially deltamethrin. Amino acids are metabolites required for protein synthesis, and have roles in metabolism as energy sources and nucleic acid precursors, they could be transformed into carbon-hydrates and fatty acids. The significant difference of amino acids between insecticide-susceptible larvae (Foshan strain) and insecticide-resistant larvae collected from geographically-close Baiyun/Yuexiu districts, was probably associated with the amino acid changes in correspondence with the deltamethrin-resistance correlated genetic changes [7] and altered expression of P450s, cuticle proteins, glutathione $S$ transferase, serine proteases, heat shock protein cytochrome s, esterase [9], as well as P450 gene SNPs such as Arg226Ser in CYP6A8, Pro175GIn in CYP9B2, His877Tyr in CYP9B2, Cys212Ser in CYP1A1 in Ae. albopictus [9]. The change of amino acids and proteins was also reported to be involved in insecticide resistance of other mosquitoes, such as arginine (ARG) changes for energy production and insecticide detoxification of Culex quinquefasciatus $L$ [19], elevated P450s and esterases (EST) in the insecticide-resistant housefly M. domestica [24], more proteins involved in oxidoreductase, transferase, hydrolase, transporter and catalytic activity, cuticle formation in the pyrethroid resistant strain of culex pipiens pallens $[25,26]$. Changes of amino acids and insect proteases also occurred under insecticide exposure for protein metabolism, such as for re-synthesis of up-regulated detoxification enzymes, suggesting a role of protein metabolism under insecticide pressure $[24,27]$.

Carnitine is derived from amino acids lysine and methionine, AcylCNs are metabolites of organic acids and beta oxidation of fats. In this study, $\mathrm{C} 0$ and $\mathrm{C} 2$ were the two most abundant AcylCNs in larvae of all five strains. The changes of free carnitine (C0) and acetyl-carnitine (C2) were regarded to be involved in energy production and insecticide detoxification of Culex quinquefasciatus $L$ [19]. In this study, five AcylCNs (C3DC, C6-1, C10, C10-1, C10-2) were found to be significantly higher in larvae from Yuexiu strain than the susceptible Foshan strain, while the levels of $\mathrm{C} 2$ were obviously lower, suggesting a probable variation of these AcylCNs in association with the development of insecticide resistance in Ae. albopictus.

\section{Conclusions}

This study first captured the characteristic of amino acids and AcyICNs profile of Ae. albopictus larvae with differential resistance to insecticides especially deltamethrin, indicating the possibility of LC-MS/MS based amino acids and AcylCNs profile as a tool for fast discrimination of deltamethrin-resistant strain from -susceptible strain of Ae. albopictus, as well as a potential way for seeking potential biomarkers for early diagnosis of insecticide resistance like deltamethrin in Ae. albopictus.

\section{Abbreviations}

AA, amino acids. AC, acylcarnitine. AcylCNs, acylcarnitine. Ae. albopictus, Aedes albopictus. PCA, principal component analysis.

\section{Declarations}

\section{Acknowledgements}

The authors would like to thank Hongxia Liu from Shanghai CDC for assistance in induction of laboratory deltamethrin-resistant strains of Ae. albopictus.

\section{Authors' contributions}

Conceptualization: Lianfen Huang, Ruili Xie, Xiao-Guang Chen, Hua Li. Data curation: Lianfen Huang, Xinhua Su, Chenfang Tang. Formal analysis: Lianfen Huang, Ruili Xie, Lilan Peng, Chenfang Tang, Jun Li, Xinhua Su. Funding acquisition: Xiao-Guang Chen, Hua Li. Investigation: Lianfen Huang, Ruili Xie, Lilan Peng, Xinhua Su. Methodology: Lianfen Huang, Ruili Xie, Lilan Peng, Chenfang Tang, Yonglan Huang, Xinhua Su. Project administration: Lianfen Huang, Ruili Xie, Jun Li. Supervision: Xiao-Guang Chen, Hua Li, Yonglan Huang. Final approval: all authors have read and approved the manuscript.

\section{Funding}

This study was supported by the National Institutes of Health, USA(R01AI083202), Guangzhou Science and technology project, China (202002030491) and the Medical Scientific Research Foundation of Guangdong Province, China (A2019512).

\section{Ethical approval and consent to participate}

Not applicable.

Consent for publication 
Not applicable.

\section{Availability of supporting data}

The insecticide resistance and Kdr mutation analysis data of the adults of Ae. albopictus from Yuexiu/Baiyun districts of Guangzhou city in 2017 is available from the paper (Su X, Guo Y, Deng J, Xu J, Zhou G, Zhou T, et al. (2019) Fast emerging insecticide resistance in Aedes albopictus in Guangzhou, China: Alarm to the dengue epidemic. PLoS Negl Trop Dis 13(9): e0007665.) at the website (https://doi.org/10.1371/journal. pntd.0007665).

\section{Competing interests}

The authors declare no conflicts of interest in this work.

\section{Authors' information}

${ }^{1}$ Department of Pathogen Biology and Experimental Teaching Center of Preventive Medicine, Guangdong Provincial Key Laboratory of Tropical Disease, School of Public Health, Southern Medical University, Guangzhou, China. ${ }^{2}$ Clinical Laboratory, Guangzhou Women and Children's Medical center, Guangzhou Medical University, Guangzhou, China. ${ }^{3}$ Newborn Screening Center, Guangzhou Women and Children's Medical center, Guangzhou Medical University, Guangzhou, China.

\section{References}

1. Manni M, Guglielmino CR, Scolari F, Vega-Rua A, Failloux AB, Somboon P, Lisa A, Savini G, Bonizzoni M, Gomulski LM, et al. Genetic evidence for a worldwide chaotic dispersion pattern of the arbovirus vector, Aedes albopictus. PLoS Negl Trop Dis. 2017;11(1):e5332.

2. Bohers C, Mousson L, Madec Y, Vazeille M, Rhim A, M'Ghirbi Y, Bouattour A, Failloux AB. The recently introduced Aedes albopictus in Tunisia has the potential to transmit chikungunya, dengue and Zika viruses. PLoS Negl Trop Dis. 2020;14(10):e8475.

3. Baldacchino F, Caputo B, Chandre F, Drago A, Della TA, Montarsi F, Rizzoli A. Control methods against invasive Aedes mosquitoes in Europe: a review. PEST MANAG SCl. 2015;71(11):1471-85.

4. Abramides GC, Roiz D, Guitart R, Quintana S, Guerrero I, Gimenez N. Effectiveness of a multiple intervention strategy for the control of the tiger mosquito (Aedes albopictus) in Spain. Trans R Soc Trop Med Hyg. 2011;105(5):281-8.

5. World Health Organization. Dengue: Guidelines for diagnosis, treatment, prevention and control: new edition. Geneva: World Health Organization; 2009.

6. World Health Organization. Global insecticide use for vector-borne disease control, a 10 year assessment (2000-2009). 2011.

7. Su X, Guo Y, Deng J, Xu J, Zhou G, Zhou T, Li Y, Zhong D, Kong L, Wang X, et al. Fast emerging insecticide resistance in Aedes albopictus in Guangzhou, China: Alarm to the dengue epidemic. PLoS Negl Trop Dis. 2019;13(9):e7665.

8. Xu J, Bonizzoni M, Zhong D, Zhou G, Cai S, Li Y, Wang X, Lo E, Lee R, Sheen R, et al. Multi-country survey revealed prevalent and novel F1534S mutation in voltage-gated sodium channel (VGSC) gene in Aedes albopictus. PLoS Negl Trop Dis. 2016;10(5):e4696.

9. Xu J, Su X, Bonizzoni M, Zhong D, Li Y, Zhou G, Nguyen H, Tong S, Yan G, Chen XG. Comparative transcriptome analysis and RNA interference reveal CYP6A8 and SNPs related to pyrethroid resistance in Aedes albopictus. PLoS Negl Trop Dis. 2018;12(11):e6828.

10. Yang T, Liu N. Genome analysis of cytochrome P450s and their expression profiles in insecticide resistant mosquitoes, Culex quinquefasciatus. PLOS ONE. 2011;6(12):e29418.

11. Lv Y, Wang W, Hong S, Lei Z, Fang F, Guo Q, Hu S, Tian M, Liu B, Zhang D, et al. Comparative transcriptome analyses of deltamethrin-susceptible and resistant Culex pipiens pallens by RNA-sEq. MOL GENET GENOMICS. 2016;291(1):309-21.

12. Zhao X, Han Q, Liu Y, Sun C, Gang X, Wang G: The Relationship between branched-chain amino acid related metabolomic signature and insulin resistance: A systematic review. J DIABETES RES 2016, 2016:2794591.

13. Chang J, Wang Q, Bhetuwal A, Liu W. Metabolic pathways underlying GATA6 regulating Trastuzumab resistance in gastric cancer cells based on untargeted metabolomics. INT J MED SCI. 2020;17(18):3146-64.

14. Nambo-Venegas R, Valdez-Vargas C, Cisneros B, Palacios-Gonzalez B, Vela-Amieva M, Ibarra-Gonzalez I, Cerecedo-Zapata CM, Martinez-Cruz E, Cortes H, Reyes-Grajeda JP, et al: Altered plasma acylcarnitines and amino acids profile in spinocerebellar ataxia type 7. Biomolecules 2020, 10(3).

15. Pomar CA, Kuda O, Kopecky J, Rombaldova M, Castro H, Pico C, Sanchez J, Palou A. Alterations in plasma acylcarnitine and amino acid profiles may indicate poor nutrition during the suckling period due to maternal intake of an unbalanced diet and may predict later metabolic dysfunction. Faseb $\mathrm{J}$. 2019;33(1):796-807.

16. Xu ZR, Zhu XY, Lu W, Sun WH, Cheng RQ, Ni JW, Xi L, Hussain K, Luo FH, Zhang MY. Altered serum amino acid and acylcarnitine profiles in hyperinsulinemic hypoglycemia and ketotic hypoglycemia. Front Endocrinol (Lausanne). 2020;11:577373.

17. Gunther SH, Khoo CM, Tai ES, Sim X, Kovalik JP, Ching J, Lee JJ, van Dam RM. Serum acylcarnitines and amino acids and risk of type 2 diabetes in a multiethnic Asian population. BMJ Open Diabetes Res Care 2020, 8(1).

18. Kasakin MF, Rogachev AD, Predtechenskaya EV, Zaigraev VJ, Koval VV, Pokrovsky AG: Changes in amino acid and acylcarnitine plasma profiles for distinguish in patients with multiple sclerosis from healthy controls. Mult Scler Int 2020, 2020:9010937.

19. Martin-Park A, Gomez-Govea MA, Lopez-Monroy B, Trevino-Alvarado VM, Torres-Sepulveda MD, Lopez-Uriarte GA, Villanueva-Segura OK, Ruiz-Herrera MD, Martinez-Fierro ML, Delgado-Enciso I, et al. Profiles of amino acids and acylcarnitines related with insecticide exposure in Culex quinquefasciatus (Say). Plos One. 2017;12(1):e169514.

20. World Health Organization. Guidelines for laboratory and field testing of mosquito larvicides. Geneva: World Health Organization; 2005. 
21. Huang LF, Li J, Peng L. The differential metabolic profiles between deltamethrin resistant and -susceptible strains of Aedes albopictus. J Med Entomol 2020.

22. World Health Organization. Test procedures for insecticide resistance monitoring in malaria vectors, bio-efficacy and persistence of insecticides on treated surfaces: report of the WHO informal consultation, Geneva, 28-30 September 1998. Geneva World Health Organization 1998.

23. Brogdon WG, McAllister JC. Simplification of adult mosquito bioassays through use of time-mortality determinations in glass bottles. J Am Mosq Control Assoc. 1998;14(2):159-64.

24. Li M, Reid WR, Zhang L, Scott JG, Gao X, Kristensen M, Liu N. A whole transcriptomal linkage analysis of gene co-regulation in insecticide resistant house flies, Musca domestica. BMC GENOMICS. 2013;14:803.

25. Lv Y, Wang W, Hong S, Lei Z, Fang F, Guo Q, Hu S, Tian M, Liu B, Zhang D, et al. Comparative transcriptome analyses of deltamethrin-susceptible and resistant Culex pipiens pallens by RNA-sEq. Mol Genet Genomics. 2016;291(1):309-21.

26. Wang W, Lv Y, Fang F, Hong S, Guo Q, Hu S, Zou F, Shi L, Lei Z, Ma K, et al. Identification of proteins associated with pyrethroid resistance by iTRAQ-based quantitative proteomic analysis in Culex pipiens pallens. Parasit Vectors. 2015;8:95.

27. Wilkins RM. Insecticide resistance and intracellular proteases. Pest Manag Sci. 2017;73(12):2403-12.

\section{Figures}

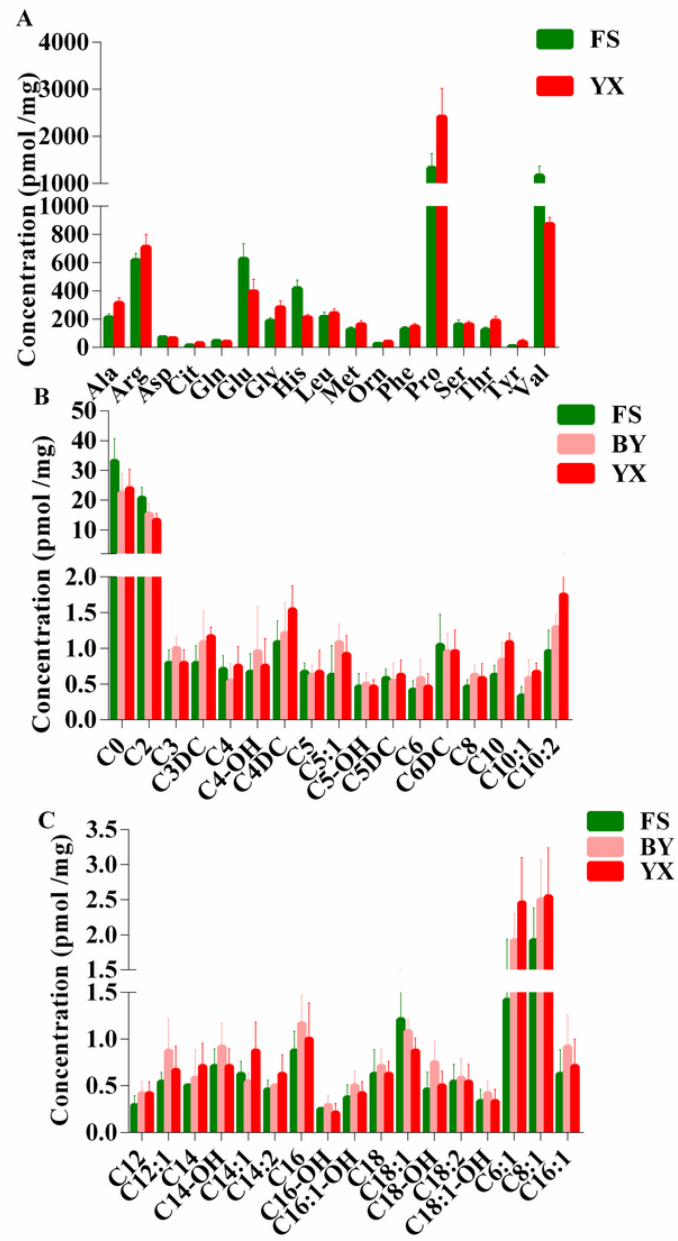

\section{Figure 1}

The concentrations of amino acids and acylcarnitines in Foshan (FS), Baiyun (BY) and Yuexiu (YX) strains 

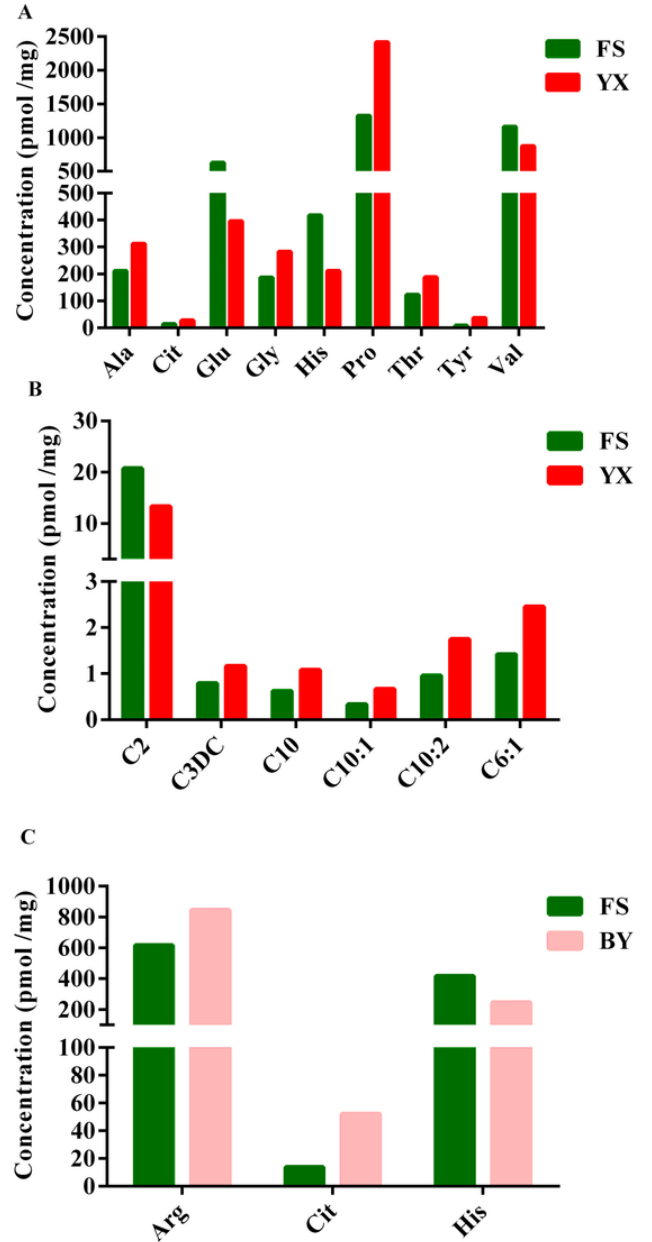

\section{Figure 2}

Differential amino acids and AcyICNss between DS-FS and DR-BY/YX larvae. (A) \& (B)significant differential amino acids and AcylCNs between susceptible Foshan (FS) and resistant Yuexiu (YX) larvae; (C) significant differential amino acids between susceptible DS-FS and Foshan (FS) and resistant Baiyun (BY) larvae. 

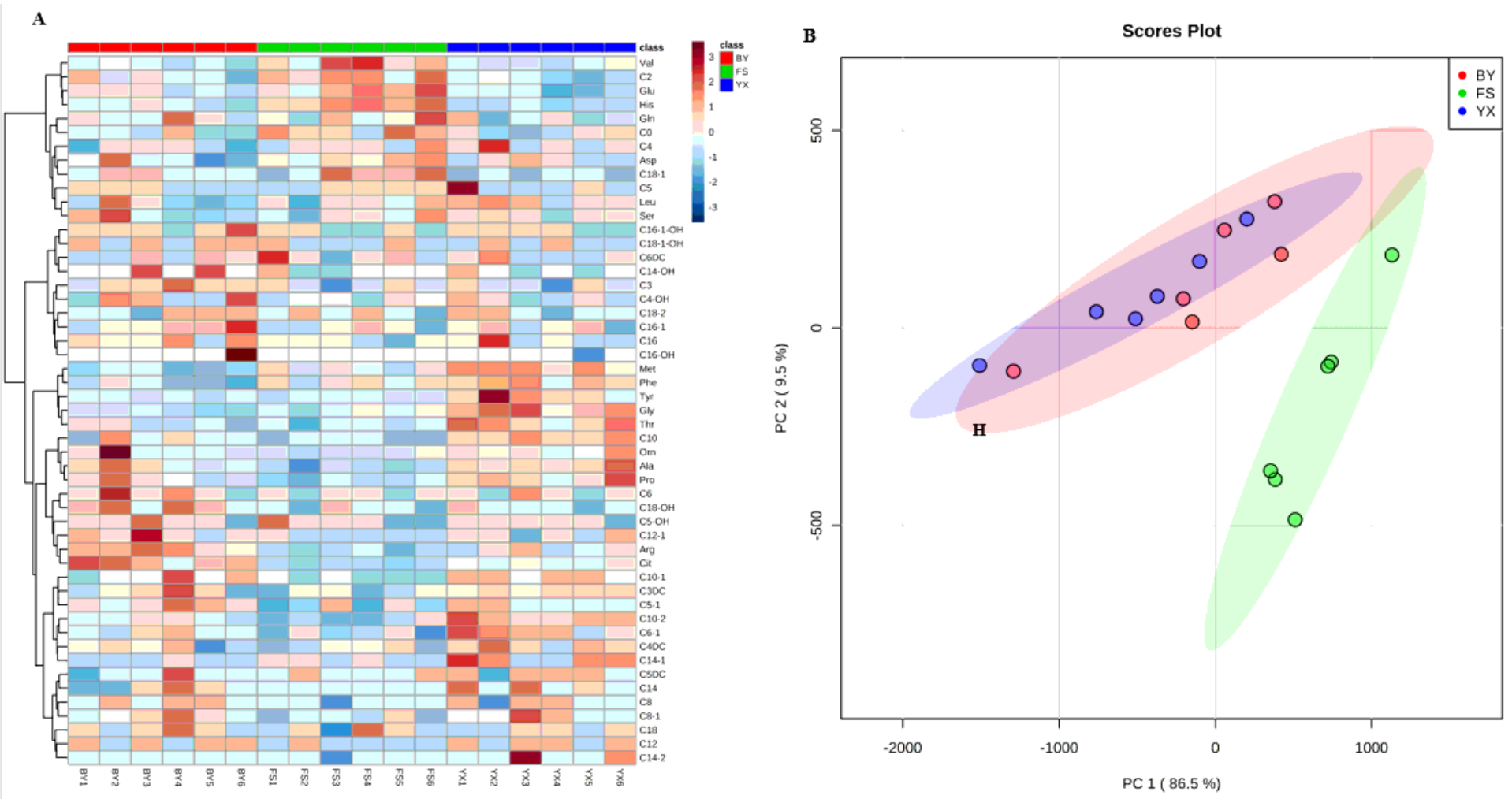

Figure 3

Heatmap (A) and PCA analysis (B) showed Differential amino acids and AcylCNs profiles between Foshan (FS)and Baiyun (BY)/Yuexiu (YX) larvae.
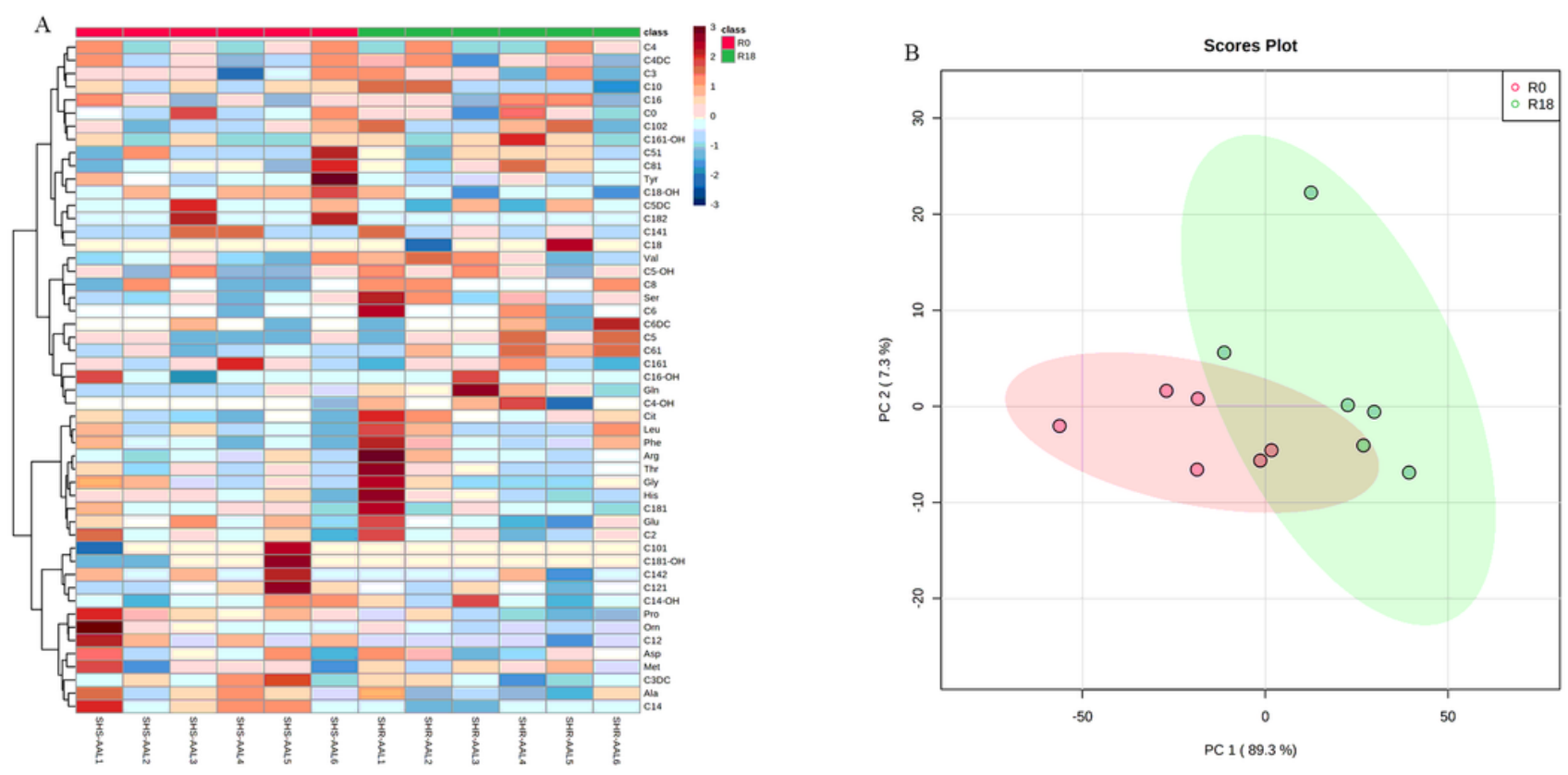

Figure 4

Heatmap (A) and PCA analysis (B) showed differential amino acids and AcylCNs profiles between larvae from deltamethrin-resistant R18 and its parental susceptible strains (RO)

\section{Supplementary Files}


This is a list of supplementary files associated with this preprint. Click to download.

- Tables1.xlsx

- Tables2.xls 